principle be separated, if $n_{r y t u p l a s m}$ can be varied independently of $n_{\text {inembram }}$, until the form component is eliminated. This requires that the methods used to vary $n_{\text {cytoplasm }}$ have no effect on the membrane spacing, structure or thickness.

Liebman et al. first recorded a birefringence spectrum of dark adapted rods in the visible region, then followed the changes in $\Delta n$ during bleaching. They found a birefringence change appearing at about the rate of the metarhodopsin $\mathrm{I} \rightarrow \mathrm{II}$ step, the 'birefringence iransient' (BRT). The figure is a schematic diagram of the fast birefringence change plotted against wavelength. This curve can be fitted by the sum of two components, one resulting from the optical effects of bleaching, and the other directly caused by changes in the membrane. The first component can be calculated theoretically, and it has the shape of the curve shown, but its position is shifted upwards so that it is centred on the axis at zero BRT. The observed change is indicated by the heavier portion of the curve. Once the effect of pigment absorption loss is accounted for, what is left is simply a constant (over the wavelength band measured) birefringence loss. This birefringence loss is attributed to a change in the intrinsic birefringence of the membrane. As in the case of nerve, there are many ways of explaining this change in terms of molecular events. It could be due to any combination of small rcorientations of lipid or protein, or slightly increased disorder. Nevertheless, Liebman et al. have detected a small change which may signal the start of the visual impulse.

Helen Saibil

\title{
Astrophysics and energy from black holes
}

EVER since Einstein's discovery of the famous equation $E=m c^{2}$ the direct conversion of mass into energy has remained man's most hopeful source of power. Atomic fission reactors, and a possible future generation of fusion devices, manage to achieve the remarkably good efficiency (by terrestrial standards) of converting about $1 \%$ of nuclear rest mass into energy. But there almost certainly lurks in the Universe a source of mass conversion with a theoretical efficiency of up to $100 \%$. This source is the famous spinning black hole.

It is now generally appreciated that black holes may only be characterised by three parameters; mass, electric charge and angular momentum. Other than this all black holes appear to the outside world to be identical, whatever their internal constitution. Black holes with rotation possess a number of strange properties which are not shared by their nonrotating counterparts. For example, suppose an inquisitive explorer were to take a trip in a rocket ship near the surface of a rotating black hole. As he approached closer, he would observe that the effect of the enormously compact mass was to try to drag his rocket around with it as it rotated. More and more fuel would need to be expended to resist this effect until, at a certain distance from the object, no amount of rocket power in the universe could prevent the ship from being dragged around in circles. The explorer has entered a new region, the ergosphere, where it is impossible to be 'at rest'. Inside the ergosphere is an event horizon, which if crossed will forbid an object to leave again. Anything which arrives inside this horizon cannot by any power available prevent itself from falling towards the centre of the black hole. But an object which has only ventured as far as the ergosphere can not only escape to the outside world again but actually do so with the bonus of more energy than it went in with. Hence the name 'ergosphere'.

The precise details of this energy-generating process were discovered by Penrose in 1969 and are based on the fact that a particle in the ergosphere is able to move along a trajectory which has negative total energy relative to a distant observer. Consequently, if a particle falls into the ergosphere and breaks into two pieces such that one of these pieces moves on a negative energy trajectory, then the other piece will re-emerge with an energy which is greater than the original combined energies of both pieces, while the sacrificed piece will plunge on into the hole, the negative energy it carries thereby reducing the total mass and angular momentum of the black hole. The process cannot, how- ever, be repeated indefinitely so that the black hole disappears altogether. Eventually, when the angular momentum has been reduced to zero, no further energy may be extracted in this way.

A rather fanciful practical application of the Penrose process has been discussed by Misner, Thorne and Wheeler (Gravitation, 908; Freeman, San Francisco, 1973). An advanced civilisation could build a city around a rotating black hole, and daily dispose of its waste by dropping it in special vessels into the ergosphere, where the waste would be tipped out along a negative energy trajectory, thereby not only obligingly disposing of itself in the most effective dustbin in the Universe, but in addition supplying the reemerging vessel with an energy equivalent of the entire waste rest mass, plus some of the rest mass of the black hole as well.

As well as contributing to the solution of cosmic ecology problems, the Penrose process might serve as a mechanism for producing high velocity jets in various astrophysical situations. A black hole at the centre of a galaxy could act as a repository for nearby stars, which might fragment appropriately on their way through the ergosphere, thereby imparting a very high velocity to one of the fragments, which would then emerge in the form of a jet.

Strict limits on the energetics of jets formed in this way have been deduced by Wald, of the University of Maryland, in the Astrophysical Journal (191, 231; 1974). The argument used involves a simple comparison of the breakup process in ordinary special relativity with that occurring in the ergosphere of a rotating black hole.

The results of this comparison are disappointing for the astrophysicist. So similar are the limiting expressions in the two cases that the energy of the emitted fragment which is attainable is not greatly different whether the black hole is there or not. For example, an object simply falling from rest a great distance away could initiate the ejection of a fragment with a velocity at most $1+v \sqrt{ } 2$ compared with the special relativistic value of $v$. The emitted fragment can never be relativistic unless the incident body or the breakup velocity are themselves relativistic.

Thus, although rotating black holes may well be responsible for triggering violent explosions at the centres of galaxies, it seems that the Penrose energy is unlikely to be making a major contribution to such events after all. 